\title{
Anatomical variants of left circumflex artery, coronary sinus and mitral valve can determine safety of percutaneous mitral annuloplasty
}

\author{
Rafał Młynarski ${ }^{1,2}$, Agnieszka Młynarska ${ }^{1,2,3}$, Maciej Sosnowski², \\ ${ }^{1}$ Department of Electrocardiology, Upper-Silesian Medical Center, Katowice, Poland \\ ${ }^{2}$ Unit of Noninvasive Cardiovascular Diagnostics, Upper-Silesian Medical Center, Katowice, Poland \\ ${ }^{3}$ Medical University of Silesia, Katowice, Poland \\ ${ }^{4} 3^{\text {rd }}$ Division of Cardiology, Medical University of Silesia, Katowice, Poland
}

\begin{abstract}
Background: The anatomical variants of the relations of the left circumflex artery $(L C x)$ and the coronary sinus (CS) determine the safety of percutaneous mitral annuloplasty (PMA) as an occlusion of the LCx and its consequences might occur in some cases. Thus, knowledge of the anatomy of cardiac anatomy and any variations are important for surgeons.

Methods: In 320 (126 women, age $59 \pm 11$ ) patients, a 64-slice computed tomography (MSCT) (Aquilion 64) was performed due to a suspicion of coronary artery disease. A scan with ECG-gating was performed using a slice thickness of $0.5 \mathrm{~mm}$ during a breath-hold. The helical pitch was 12.8, rotation time: $0.4 \mathrm{~s}$ and average tube voltage: $135 \mathrm{kV}$ at $380 \mathrm{~mA}$. $100 \mathrm{~mL}$ of non-ionic contrast agent was given in three phases at an average rate of $4.5 \mathrm{~mL} / \mathrm{s}$. In each case, ten $3 D$ volume rendering and $2 D$ multiplanar reconstructions of the vessels were created (Vitrea 2).
\end{abstract}

Results: The CS was visualized in all cases and the LCX in 315 (98.4\%). In 302 (95.8\%) cases, the CS was the dominant vessel; in 10 (3.17\%) cases both vessels were equal and the LCX was dominant in only $3(0.9 \%)$ cases. 52 anatomical variants were identified; 3 of them were the most common (in 164/315 cases; 50.8\%). The CS usually lies above the atrioventricular (AV) sulcus (239-75.9\%) and the LCx within the AV sulcus (173-54.9\%). In 235 (74.6\%) cases, the LCX was closer (than the CS) to the mitral valve. It was found that in $78(24.7 \%)$ cases, the LCX was beneath the CS in selected phases (a risk of LCX occlusion by a PMA device). The LCx closer to the mitral valve, which is considered as a safe feature for PMA, was observed in only 75 (23.8\%) of the cases. The most dangerous pattern was found in 19 cases (6.1\%) - 2 or 3 CS/LCx crosses.

Conclusions: The huge anatomical variability of the anatomy of the CS/LCX strengthens the role of MSCT before PMA implantation. (Cardiol J 2013; 20, 3: 235-240)

Key words: coronary vessels, anatomical variants, computed tomography

\section{Introduction}

Percutaneous mitral annuloplasty (PMA) can be an alternative method of treatment for patients with ischemic mitral regurgitation $[1,2]$. In this method, the circumference of the mitral annulus is reduced in the antero-posterior diameter by a dedicated device such as e.g. a CARILLON (Cardiac Dimensions, USA). Devices are implanted percutaneously via the coronary sinus (CS) ostium

Address for correspondence: Agnieszka Młynarska, MS, PhD, Upper-Silesian Medical Center, Unit of Noninvasive Cardiovascular Diagnostics, ul. Ziołowa 45/47, Katowice, Poland, fax: +48 3225240 98, e-mail: agnieszkawlodyka@o2.pl 
from the right atrium and finally into the CS and great cardiac vein. It usually consists of two parts - the proximal part in the CS ostium and the distal part in the distal great cardiac vein $[1,3]$. The two parts are connected by a "bridge" that reduces the circumference of the mitral annulus. The results of a CARILLON Mitral Annuloplasty Device European Union Study (AMADEUS) proved that using the CARILLON is feasible in patients with heart failure (HF) and is associated with a low incidence of major adverse events and is associated with an improvement in quality of life and exercise tolerance [4]. The latest study - TITAN (Transcatheter Implantation of Carillon Mitral Annuloplasty Device) proved that percutaneous CS-based mitral annuloplasty can significantly and safely reduce functional mitral regurgitation in $\mathrm{HF}$ patients. It was also documented that left ventricle reverse remodeling occurred over 12 months and that improved measures of clinical outcome occurred over 24 months [5].

The anatomical variants of relations of the left circumflex artery (LCx) and the CS determine the safety of PMA as an occlusion of the LCx and its consequences might occur in some cases. Thus, knowledge of the anatomy of the cardiac vessels and any variations are important for surgeons. Multi-slice computed tomography (MSCT) allows the visualization of the relationships between the mitral valve (MV), the $\mathrm{LCx}$ and the CS so that the risk of occluding the $\mathrm{LCx}$ can be minimized $[3,6]$. In our previous study, we documented how to visualize the LCx and CS in MSCT [6]. However, there is still the question of which anatomical variants $\mathrm{CS} / \mathrm{LCx} / \mathrm{MV}$ can be met by PMA operators. In this study, we used our previous experience to describe the anatomy of the relations of the structures of the heart that are necessary for PMA and found anatomical variants of the $\mathrm{CS} / \mathrm{LCx}$ with the mitral valve.

\section{Methods}

The data for 320 consecutive patients (126 women, aged $59 \pm 11$ ) from 64 -slice computed tomography (Aquilion 64, Toshiba Medical, Japan) was retrospectively analyzed in order to evaluate the CS, LCx and MV annulus. MSCTs of the heart were originally performed due to a suspicion of coronary artery disease (CAD). Patients were excluded if the image quality was not diagnostic for the research purpose.

The local Ethics Committee approved the study protocol. The study protocol conformed to the version of the Helsinki Convention that was current at the time the study was designed.

\section{Computed tomography procedure}

Scanning with retrospective ECG-gating was performed during a breath-hold using 64 slices with a collimated slice thickness of $0.5 \mathrm{~mm}$. A breath-hold examination was performed in order to adjust the scanner settings. The helical pitch was 12.8 (best mode) and the rotation time was $0.4 \mathrm{~s}$. The tube voltage was strictly dependent on the patient's body mass index (BMI): for BMI $<23.9$ it was $120 \mathrm{kV}$ at $330 \mathrm{~mA}$, for $\mathrm{BMI}=24.0-29.9$ it was $135 \mathrm{kV}$ at $380 \mathrm{~mA}$ and for BMI $>30.0$ it was $135 \mathrm{kV}$ at $430 \mathrm{~mA}$. We used a pre-selected region of interest (ROI) in the descending aorta. Triggering started at 180 Hounsfield units. On average, $100 \mathrm{~mL}$ of non-ionic contrast agent (Ioperamid, Ultravist 370, Schering, Germany) was given to each patient during the examination at an average rate of $4.5 \mathrm{~mL} / \mathrm{s}$. The contrast agent was given in three phases: $90 \mathrm{~mL}$ of contrast agent (average), then $24 \mathrm{~mL}$ of contrast agent followed by $16 \mathrm{~mL}$ of saline flush $(60 \% / 40 \%)$ and finally $30 \mathrm{~mL}$ of saline. The cut-off for heart rate (HR) was set at $65 \mathrm{bpm}$. If the HR was higher, metoprolol succinate (Betaloc, Astra Zeneca, Sweden) at a dosage of 5-10 mg was given intravenously, unless contraindicated. If the expected HR slowing did not occur, the patient was excluded from the study. Sublingual nitroglycerin was not given before image acquisition.

\section{Computed tomography reconstruction}

Reconstructions of the data were performed retrospectively on Vitrea 2 workstations (Vital Images, USA; software version 3.9.0.0 and 5.1). In each case, we created ten axial image series as well as $3 \mathrm{D}$ volume rendering (VR) reconstructions from $0 \%$ to $90 \%$ R-R intervals (step 10\%) using a $2.0 \mathrm{~mm}$ slice thickness in order to reduce the large amount of data. Earlier publications including studies from our unit have sho wn that the phase of the RR interval at which reconstructions are performed is an important factor that affects the quality of the visualization [6-9]. This is especially important when vessels other than coronary arteries need to be analyzed. Based on the results from our previous paper, thanks to retrospective gating, reconstructions of the parallel visualization of both the $\mathrm{LCx}$ and the $\mathrm{CS}$ to determine the relations between these vessels was performed in the 70-80\% RR phase. An additional independent reconstruction that is optimal for the LCx (70-80\% RR) as well as the CS (30-40-50\% $\mathrm{RR}$ ) was also performed. The dominant vessel was judged to be the larger vessel. 
Table 1. Average values (min, max, SD) of the main cardiac function parameters for the patients included.

\begin{tabular}{lcccc}
\hline & Average value & Min. value & Max. value & SD \\
\hline Ejection fraction [\%] & 62.0 & 8 & 86 & \pm 10.48 \\
End-diastolic volume $[\mathrm{mL}]$ & 145.42 & 69 & 464 & \pm 44.69 \\
End-systolic volume [mL] & 58.43 & 11 & 425 & \pm 37.82 \\
Stroke volume [mL] & 87.18 & 39 & 151 & \pm 20.11 \\
Cardiac output [L/min] & 5.38 & 1,8 & 13,9 & \pm 1.54 \\
Myocardial mass $[\mathrm{g}]$ & 140.91 & 60 & 371 & \pm 45.37 \\
Myocardial volume [mL] & 131.91 & 13 & 352 & \pm 43.57 \\
Heart rate [bpm] & 61.54 & 20 & 157 & \pm 10.36 \\
\hline
\end{tabular}

\section{Results}

The hemodynamic characteristics of the patients are presented in Table 1.

Anatomy of the heart in cardiac computed tomography is presented in the Figure 1 . The CS was visualized in all cases and the circumflex branch of the left coronary artery in $315(98.4 \%)$ cases. In $302(95.8 \%)$ cases, the CS was the dominant vessel, in $10(3.2 \%)$ both vessels were equal and the $\mathrm{LCx}$ was dominant in only $3(0.9 \%)$ cases.

The CS was usually above the atrio-ventricular (AV) sulcus in the atrial direction (239 cases; $75.9 \%)$, in $72(22.8 \%)$ cases it was under and in $4(1.3 \%)$ cases it was in the sulcus. The LCx was usually within the AV sulcus (173 cases; $54.9 \%$ ), in 67 cases below it (21.3\%) and in 66 cases above (20.9\%) it. In $9(2.9 \%)$ cases, it was not possible to evaluate the LCx. In tomographic images, the AV sulcus should be identified with the MV annulus. In $235(74.6 \%)$ cases, the LCx was closer (than the $\mathrm{CS}$ ) to the mitral (Fig. 2A).

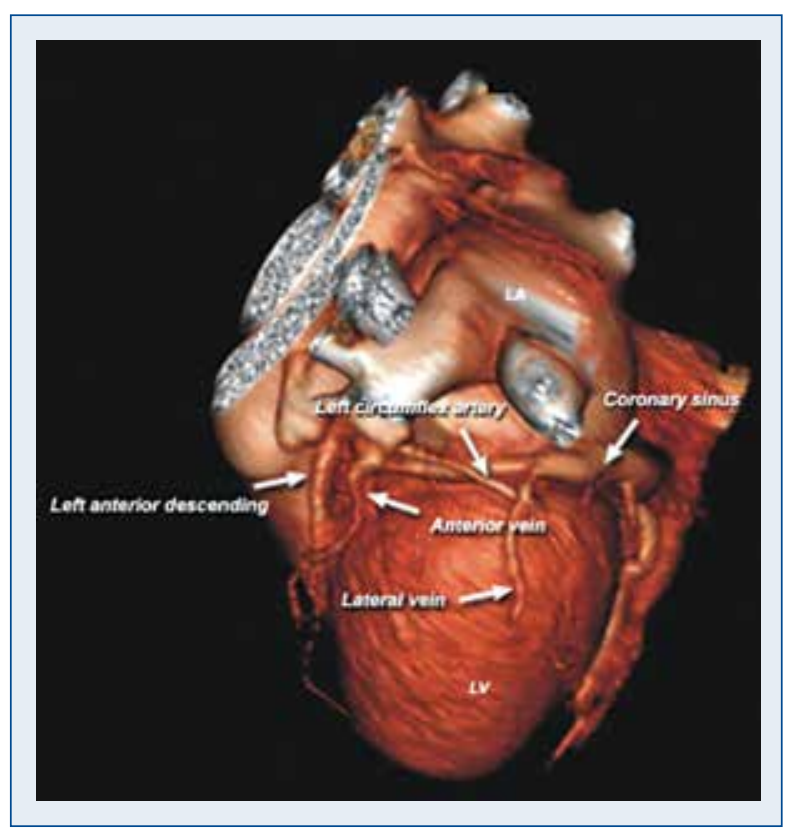

Figure 1. Anatomy of the heart in cardiac computed tomography - 3DVR; LA — left atrium; LV — left ventricle.

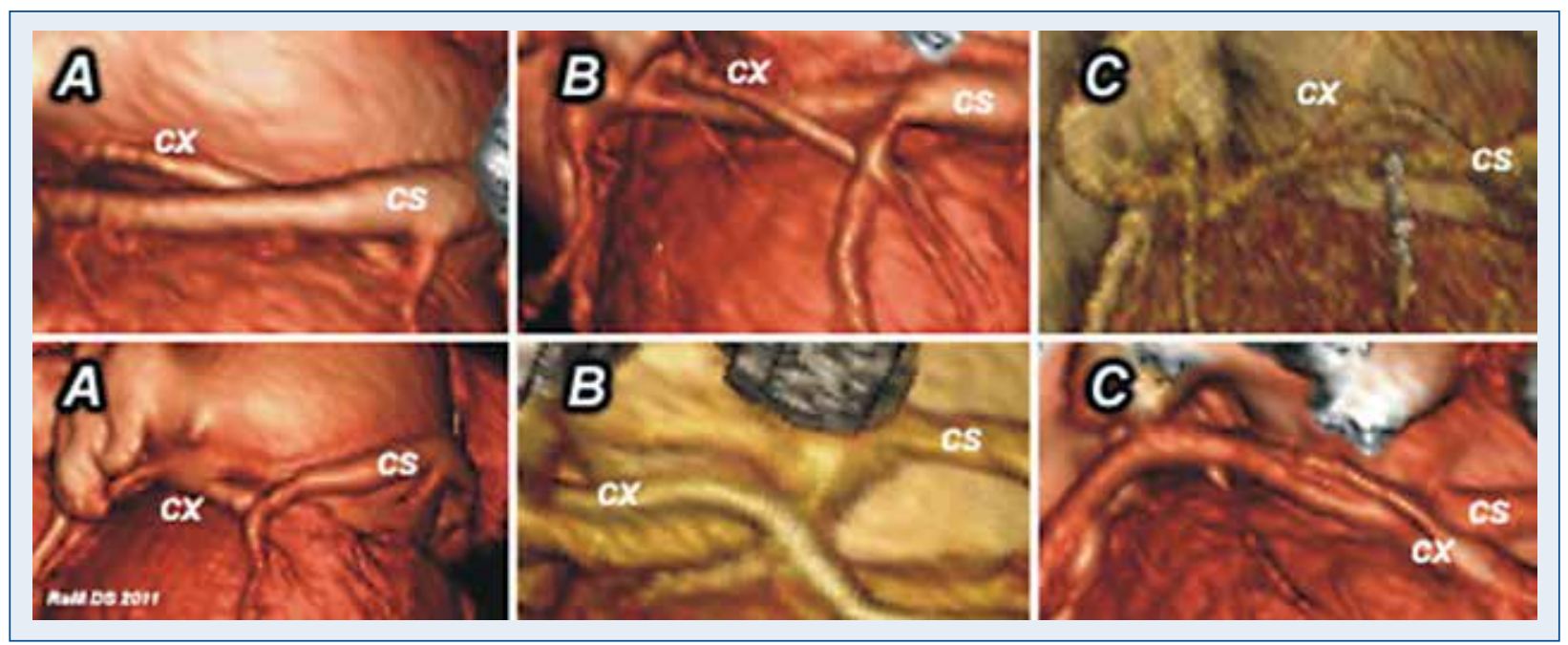

Figure 2. Examples of anatomical variants coronary sinus (CS)/left circumflex (CX) in cardiac computed tomography - 3DVR. 
Table 2. The most common anatomical variants found in this study.

\begin{tabular}{|c|c|c|c|c|c|c|c|}
\hline \multirow[t]{2}{*}{ No. } & \multirow{2}{*}{$\begin{array}{c}\text { Dominant } \\
\text { vessel }\end{array}$} & \multicolumn{2}{|c|}{ Localization } & \multirow{2}{*}{$\begin{array}{l}\text { Vessel } \\
\text { higher }\end{array}$} & \multirow{2}{*}{$\begin{array}{l}\text { Number } \\
\text { of crosses }\end{array}$} & \multirow{2}{*}{$\begin{array}{l}\text { Vessels close } \\
\text { to the sulcus }\end{array}$} & \multirow{2}{*}{$\begin{array}{c}\text { Frequency } \\
\text { cases } / \%\end{array}$} \\
\hline & & CS & LCx & & & & \\
\hline 1 & CS & $\begin{array}{l}\text { Above } \\
\text { sulcus }\end{array}$ & In sulcus & $\mathrm{CS}$ & 1 & LCx & $107 / 33.9$ \\
\hline 2 & CS & $\begin{array}{l}\text { Above } \\
\text { sulcus }\end{array}$ & $\begin{array}{l}\text { Beneath } \\
\text { sulcus }\end{array}$ & CS & 1 & LCx & $30 / 9.5$ \\
\hline 3 & $\mathrm{CS}$ & $\begin{array}{l}\text { Above } \\
\text { sulcus }\end{array}$ & $\begin{array}{l}\text { Above } \\
\text { sulcus }\end{array}$ & $\mathrm{CS}$ & 1 & LCx & $27 / 8.6$ \\
\hline 4 & $\mathrm{CS}$ & In sulcus & In sulcus & CS & 1 & LCx & $15 / 4.8$ \\
\hline 5 & CS & $\begin{array}{l}\text { Above } \\
\text { sulcus }\end{array}$ & In sulcus & CS & 1 & CS & $11 / 3.5$ \\
\hline 6 & CS & $\begin{array}{l}\text { Above } \\
\text { sulcus }\end{array}$ & $\begin{array}{l}\text { Beneath } \\
\text { sulcus }\end{array}$ & $\mathrm{CS}$ & 1 & CS & $10 / 3.2$ \\
\hline
\end{tabular}

CS - coronary sinus; LCX - circumflex artery

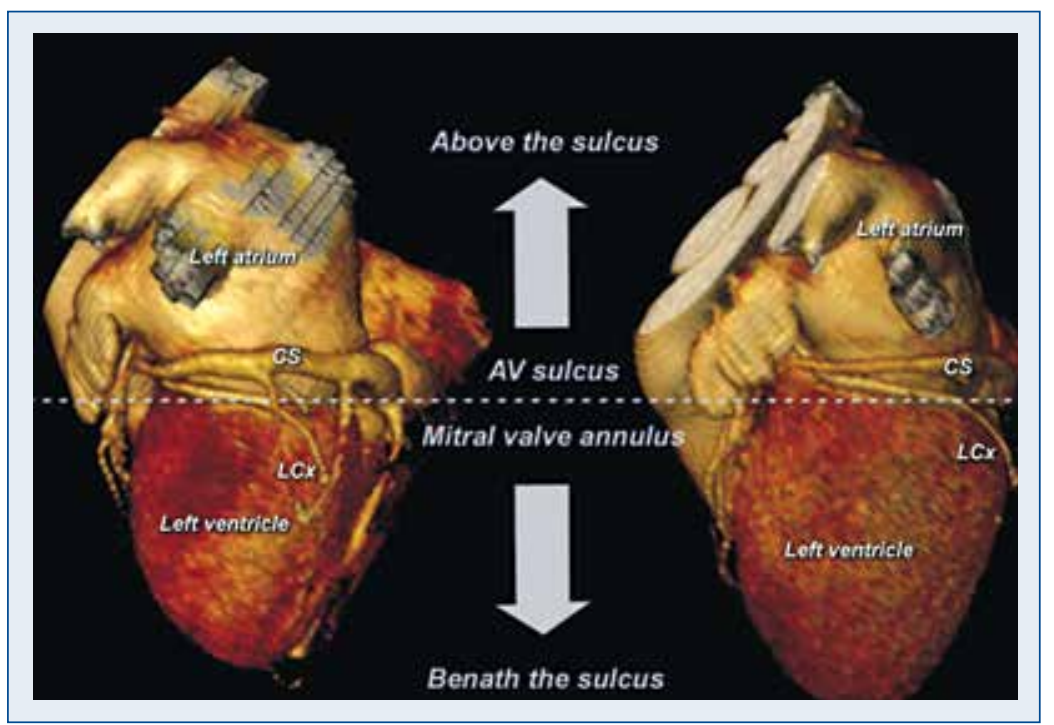

Figure 3. The most common variant of coronary sinus (CS)/left circumflex (LCx) artery in cardiac computed tomography - 3DVR.

In $78(24.7 \%)$ cases, the LCx entered beneath the CS in selected phases (not all). In those patients there was risk of $\mathrm{LCx}$ occlusion by a PMA device. The LCx running closer to the MV, which is considered as a safe feature for PMA, was observed in only $75(23,8 \%$ ) cases (Fig. 2B). The most dangerous pattern where 2 or $3 \mathrm{CS} / \mathrm{LCx}$ crosses occurred was found in 19 (6.1\%) cases (Fig. 2C).

It was possible to identify 52 anatomical variants in the examined population. Three of them are the most common (they were identified in 164 of the 315 cases; $50.8 \%$ ). The 6 most frequent variants are presented in Table 2 . In the most frequent anatomical variant, the CS lies above the
AV sulcus and the LCx in the AV sulcus and can potentially cause problems during the implantation of PMA devices. In this variant, there is one cross $\mathrm{CS} / \mathrm{LCx}$ and vessel close to the sulcus is $\mathrm{LCx}$. This variant is presented in Figure 3.

\section{Discussion}

The safety and usefulness of PMA has been confirmed in human studies such as e.g. AMADEUS and lately the TITAN trials $[4,5,10]$. In 48 patients enrolled in the AMADEUS trial the major adverse event rate was $13 \%$ at 30 days [4]. At 6 months, the degree of mitral regurgitation 
reduction ranged from $22 \%$ to $32 \%$. Six-minute walk distance improved from $307 \pm 87 \mathrm{~m}$ to $403 \pm$ $\pm 137 \mathrm{~m}$ at 6 months $(\mathrm{p}<0.001)$. Quality of life also statistically improved ( $\mathrm{p}<0.001)$.

In the latest PMA study — TITAN study the 30-day major adverse event rate was $1.9 \%$ [5]. Reductions of mitral regurgitation represented by regurgitant volume was observed $(34.5 \pm$ $\pm 11.5 \mathrm{~mL}$ to $17.4 \pm 12.4 \mathrm{~mL}$ at 12 months; $\mathrm{p}<0.001)$. Authors concluded that percutaneous reduction of mitral regurgitation using a CS approach is associated with reverse left ventricular remodeling.

One of the important factors that determine the success of a procedure is to avoid LCx compression by a PMA device. This strictly depends on the anatomical relationships between the $\mathrm{LCx}$ and the CS. MSCT can precisely document the anatomy of the coronary arteries and coronary veins $[3,6]$; however, this knowledge should be confirmed by anatomo-pathological studies.

In 2006 Maselli et al. [11] published the results of an anatomical study of the human CS in relation to the MV and coronary arteries in 61 excised cadaveric human hearts. They found that the $\mathrm{LCx}$ lies between the CS and the MV annulus in $63.9 \%$ - in our study it was $74.6 \%$.

In 2007 Tops et al. [12] analyzed 105 consecutive patients referred for MSCT coronary angiography. The patients were divided into 3 groups according to the presence of $\mathrm{CAD}$ and $\mathrm{HF}$. They concluded that the LCx, CS and MV can be analyzed in MSCT. This paper is relevant to our study as they used the same MSCT scanner with retrospective gating. They found that in $68 \%$ of the patients, the $\mathrm{LCx}$ coursed between the CS and the mitral annulus - in our study it was $74.6 \%$. At the level of the MV annulus, the CS was located more superiorly in $90 \%$ in their study - in our study $72.8 \%$.

In the next paper from 2008, Plass et al. [13] analyzed the relations of the $\mathrm{LCx} / \mathrm{CS}$ in normal and insufficient MV. In this research, the CS was usually (in 34\%) positioned at the same level as the MV and in $54 \%$ of the cases, it was above the annulus. Those results are similar to our results $-75.8 \%$ and $21.3 \%$, respectively. Swiss authors also found that in $33 \%$ of patients, the LCx ran under the CS - in our research it was $24.7 \%$. Cited paper is one of the first analyses of $\mathrm{CS} / \mathrm{LCx}$ relations using MSCT; however, the examined group is rather small when compared to our study (50 to 320 patients).

Mao et al. [14] examined 231 patients using electron-beam computed tomographic angiography (EBCTA) - they concluded that EBCTA can pro- vide a $3-D$ visualization of most of the components of the coronary venous system in relation to the coronary arteries. They also found that in $80.8 \%$ of the cases, the LCx lies deeper than the CS, which is consistent with our results.

The authors cited above did not try to find any anatomical variants and only relations were examined and the results of those relations are comparable to ours. To the best of our knowledge, our paper is the first, large-scale study (320 patients included) where anatomical variants, the $\mathrm{CS} / \mathrm{LCx} /$ $/ \mathrm{MV}$, are presented.

Fortunately, recently published results about the implantation of PMA devices have proved that the number of problems is smaller than the anatomy of the key vessels from the cited researches have shown. In the TITAN study, only two myocardial infarctions were found during follow-up in the implanted cohort and analysis showed that neither were PMA device related [5].

In our opinion, problems can also be caused by dynamic changes (the LCx moving in relation to the CS). This thesis is only based on observation, to date, it has not been scientifically proved; however, it seems that in different phases of the cardiac cycle, the anatomical relations between both vessels can change significantly.

Further clinical research in PMA implantation will evaluate any potential problems and clinical pitfalls. Data from this paper should be made available to people performing PMA implantations. There are no simple transpositions of anatomical findings (either anatomical or tomographic) into problems or accidental LCx occlusion that might occur during PMA implantation.

It is necessary to mention that there are also another methods which can reduce mitral regurgitation and are not anatomy CS/MV dependent. An example of such system is the MitraClip (Abbott, USA) which is a catheter-based less-invasive therapy for MV repair. To access the MV, the guide catheter is inserted into the femoral vein to reach the heart and deploys the MitraClip device. In latest research overall MitraClip procedural success rate was $96.5 \%$ with an in-hospital mortality rate of $3.5 \%$ [15]. Next few years will show which methods will get more outcomes for mitral regurgitation patients.

\section{Limitations of the study}

This study is a descriptive anatomical study. At the time of its publication, the Authors have no practical experience with PMA. We believe that the experience of surgeons is a critical factor in the success of the procedure. 


\section{Conclusions}

It is possible to visualize the CS, the circumflex branch of the left coronary artery and the MV annulus using cardiac computed tomography. The huge anatomical variability of the anatomy of the $\mathrm{CS}$ and the circumflex branch of the left coronary artery strengthens the potential role of performing computed tomography before PMA. In the most frequent anatomical variant, the CS lies above the $\mathrm{AV}$ sulcus and the LCx in the AV sulcus and can potentially cause problems during the implantation of PMA devices.

\section{Acknowledgments}

The authors are indebted to the radiology technologists for their technical support.

\section{Conflict of interest: none declared}

\section{References}

1. Masson JB, Webb JG. Percutaneous mitral annuloplasty. Coron Artery Dis, 2009; 20: 183-188.

2. Johnston DR, Gillinov AM, Blackstone EH et al. Surgical repair of posterior mitral valve prolapse: implications for guidelines and percutaneous repair. Ann Thorac Surg, 2010; 89: 1385-1394.

3. Gopal A, Shah A, Shareghi Set al. The role of cardiovascular computed tomographic angiography for coronary sinus mitral annuloplasty. J Invasive Cardiol, 2010; 22: 67-73.

4. Schofer J, Siminiak T, Haude M et al. Percutaneous mitral annuloplasty for functional mitral regurgitation: Results of the CARILLON Mitral Annuloplasty Device European Union Study. Circulation, 2009; 120: 326-333.

5. Siminiak T, Wu JC, Haude M et al. Treatment of functional mitral regurgitation by percutaneous annuloplasty: results of the TITAN Trial. Eur J Heart Fail, 2012; 14: 931-938.
6. Mlynarski R, Mlynarska A, Wilczek J, Sosnowski M. Optimal visualization of heart vessels before percutaneous mitral annuloplasty. Cardiol J, 2012; 19: 459-465.

7. Mlynarski R, Sosnowski M, Wlodyka A, Chromik K, Kargul W, Tendera M. Optimal image reconstruction intervals for noninvasive visualization of the cardiac venous system with a 64 -slice computed tomography. Int J Cardiovasc Imag, 2009; 25: 635-641 .

8. Mlynarska A, Mlynarski R, Kargul W, Sosnowski M. Quality of visualization of coronary venous system in 64-slice computed tomography. Cardiol J, 2011; 18: 146-150.

9. Mlynarski R, Sosnowski M, Wlodyka A, Kargul W, Tendera M. A user-friendly method of cardiac venous system visualization in 64-slice computed tomography. Pacing Clin Electrophysiol, 2009; 32: 323-329.

10. Siminiak T, Hoppe UC, Schofer J et al. Effectiveness and safety of percutaneous coronary sinus-based mitral valve repair in patients with dilated cardiomyopathy (from the AMADEUS trial). Am J Cardiol, 2009; 104: 565-570.

11. MaselliD, GuarracinoF, ChiaramontiF,MangiaF,Borelli G, MinzioniG. Percutaneous mitral annuloplasty: an anatomic study of human coronary sinus and its relation with mitral valve annulus and coronary arteries. Circulation, 2006; 114: 377-380.

12. Tops LF, Van de Veire NR, Schuijf JD et al. Noninvasive evaluation of coronary sinus anatomy and its relation to the mitral valve annulus: implications for percutaneous mitral annuloplasty. Circulation, 2007; 115: 1426-1432.

13. Plass A, Valenta I, Gaemperli O et al. Assessment of coronary sinus anatomy between normal and insufficient mitral valves by multi-slice computer tomography for mitral annuloplasty device implantation. Eur J Cardiothorac Surg, 2008; 33: 583-589.

14. Mao S, Shinbane JS, Girsky MJ et al. Coronary venous imaging with electron beam computed tomographic angiography: Three-dimensional mapping and relationship with coronary arteries. Am Heart J, 2005; 150: 315-322.

15. Paranskaya L, D’Ancona G, Bozdag-Turan I et al. Early and mid-term outcomes of percutaneous mitral valve repair with the MitraClip ${ }^{\circledR}$ : comparative analysis of different EuroSCORE strata. EuroIntervention, 2012; 8: 571-578. 